Théologiques

Théologiques

\title{
L'attrait de mouvements ultra-orthodoxes parmi les jeunes Juifs d'origine marocaine à Montréal. Échos diasporiques et constructions locales
}

\section{Jean-Luc Bédard}

Volume 15, numéro 1, 2007

Mémoires et usages religieux de l'espace

URI : https://id.erudit.org/iderudit/017644ar

DOI : https://doi.org/10.7202/017644ar

Aller au sommaire du numéro

Éditeur(s)

Faculté de théologie et de sciences des religions, Université de Montréal

ISSN

1188-7109 (imprimé)

1492-1413 (numérique)

Découvrir la revue

Citer cet article

Bédard, J.-L. (2007). L'attrait de mouvements ultra-orthodoxes parmi les jeunes Juifs d'origine marocaine à Montréal. Échos diasporiques et constructions locales. Théologiques, 15(1), 131-150. https://doi.org/10.7202/017644ar
Résumé de l'article

Mon analyse porte sur des constructions de la mémoire et de l'identité, parmi trois générations de Juifs montréalais d'origine marocaine. En particulier, les rapports avec les mouvements ultra-orthodoxes sont révélateurs de positions quant à la judéité et à la séphardité, dans un processus de différenciation par rapport aux coreligionnaires ashkénazes. À travers une analyse du rapport au passé et des constructions de la mémoire, en plus de l'historiographie des rapports entre Ashkénazes et Sépharades depuis l'expulsion d'Espagne de 1492, il en découle une remise en question, partielle, de ces deux catégories ethnoreligieuses. Comme les cadres sociaux de la mémoire, la notion de frontière permet d'éclairer les processus contemporains de représentation de soi et des autres, parmi cet ensemble diasporique qui négocie ainsi, à la fois, ses rapports à la société locale, ses frontières internes et ses dynamiques transnationales.
Tous droits réservés (c) Faculté de théologie et de sciences des religions, Université de Montréal, 2007
Ce document est protégé par la loi sur le droit d'auteur. L'utilisation des services d’Érudit (y compris la reproduction) est assujettie à sa politique d'utilisation que vous pouvez consulter en ligne.

https://apropos.erudit.org/fr/usagers/politique-dutilisation/ 


\title{
L'attrait de mouvements ultra-orthodoxes parmi les jeunes Juifs d'origine marocaine à Montréal. Échos diasporiques et constructions locales
}

\author{
Jean-Luc BÉDARD \\ Anthropologue, chercheur autonome
}

Montréal

À l'instar d'autres diasporas contemporaines, la diaspora judéo-marocaine à Montréal semble être structurée de façon chaordique. Ce néologisme, proposé par Werbner (2001), conjoint une évolution chaotique, où l'hétérogénéité croît et se complexifie, avec le maintien d'éléments identitaires demeurés en partage. On trouve en effet, chez les Juifs montréalais d'origine marocaine, un sentiment de coresponsabilité à l'égard de la situation dans le pays d'accueil et, bien sûr, en Israël. Toutefois, au-delà de ce sentiment, on peut chercher longtemps ce qui est partagé par l'ensemble du groupe diasporique. Ceci découle du fait que celui-ci est traversé par une vaste diversité, autant en termes sociodémographiques (scolarité, occupations, parcours migratoire, religiosité, langues maternelles et d'usage, etc.) qu'en ce qui touche les critères habituellement retenus pour définir une diaspora - en particulier, le rapport à une terre d'origine et le maintien de frontières (Brubaker 2005). L'hétérogénéité des judaïcités montréalaises d'origine marocaine couvre donc un spectre très large de pratiques et de positions, tant sur les plans religieux, culturels qu'économiques. Si on s'en tient à la seule pratique religieuse, on trouve des individus épousant un athéisme doublé d'un anticléricalisme affirmé, tandis que d'autres, parfois même unis aux premiers par des liens familiaux étroits, répondent à l'appel de mouvements ultra-orthodoxes, en soi un courant hétérogène! Il ne faudrait pas oublier, dans ce survol rapide, la majorité qui se déclare " orthodoxe », non sans susciter certaines ambiguïtés, particulièrement en 
contexte montréalais ${ }^{1}$. Ainsi, du fait de cette hétérogénéité, la description des quelque 18000 Juifs montréalais d'origine marocaine nécessite plusieurs nuances et mises au point, comme nous le verrons plus loin, afin d'éviter des généralisations réductrices.

Cet article se penche sur des éléments de discours et de pratiques identitaires relevés chez de jeunes adultes d'origine marocaine, touchant les rapports avec l'ultra-orthodoxie. Les données ont été amassées durant mon terrain de recherche doctorale à Montréal, entre 2000 et 2002. Si certains se disent attirés par les mouvements ultra-orthodoxes, d'autres expriment des réserves, voire des positions critiques à leur égard, tandis que d'autres encore, plus minoritaires, se situent en périphérie. La judéité chez ces derniers se décrit par des références culturelles et symboliques, plutôt que religieuses au sens strict (c'est-à-dire par l'entremise de pratiques ritualisées et institutionnalisées). Par l'analyse des constructions de la mémoire, je tente d'indiquer comment le contexte social actuel — aussi bien en Israël, au Maroc qu'au Canada et dans les autres lieux de la diaspora - contribue à un recadrage d'importantes catégories à l'intérieur du milieu juif, jusqu'ici fondées sur les ethnonymes «ashkénazes » et "sépharades ». Dans certains cas, j'ajouterai la toile de fond des constructions mémorielles mentionnées par leurs parents et grands-parents. L'analyse permettra de situer la nature du recadrage de ces catégories, prenant forme dans le contexte contemporain, radicalement différent de celui qui prévalait jusqu'à la moitié du $\mathrm{XX}^{\mathrm{e}}$ siècle. Ce sera aussi l'occasion de commenter l'analyse de la modernité proposée par Bauman (2000). Celui-ci avance que la période actuelle serait celle de la modernité liquide, où identités et appartenances sont caractérisées par la fluidité des liens sociaux, traversant toutes les sphères de la vie (famille, travail, etc.). Le sens accordé à ces liens en ressort radicalement transformé, comme son analyse subséquente (2004) le démontre encore davantage. On peut toutefois se demander si cette analyse

1. C'est ainsi, " orthodoxe ", qu'est aussi nommée une branche du judaïsme issue des judaïcités européennes, ou ashkénazes, découlant des divisions apparues dès le XVIII siècle, particulièrement en Allemagne. Les Juifs montréalais d'origine marocaine se désignant ainsi sont parfois assimilés à ces Juifs ashkénazes dans des documents statistiques, ce qui brouille considérablement le portrait. Par ailleurs, certains Sépharades d'origine marocaine "sympathisent" avec des mouvements hassidiques (Gutwirth 2004), sans pour autant joindre leurs rangs. Enfin, dans la direction plus libérale du spectre, d'autres mentionnent qu'ils souscrivent à un judaïsme « orthodoxe» tout en ne pratiquant qu'occasionnellement, faisant partie des "Juifs de Kippour », ne visitant la synagogue qu'aux grandes occasions (fêtes, bar-mitsvah, etc.). L'orthodoxie est donc utilisée pour désigner des réalités très variées. 
de la modernité vaut également pour des groupes diasporiques, minoritaires dans leur société d'accueil, traversés par des liens transnationaux et d'importantes subdivisions internes.

\section{Premier constat: une diversification croissante, voire antinomique}

Depuis les années 1990, une partie des jeunes Juifs montréalais d'origine marocaine s'est rapprochée de certaines mouvances ultra-orthodoxes, tandis que d'autres resituent leur pratique dans un giron «traditionnel ${ }^{2}$ ». Par ailleurs, d'autres jeunes adultes affichent une judéité pratiquement détachée de références religieuses. Dans ces familles, la plupart se situent alors dans un prolongement consacrant le parcours des générations ascendantes, caractérisé par un éloignement graduel des pratiques religieuses. Dans la première partie de cet article, je tenterai d'illustrer ce mouvement quasi antinomique, à partir des données de ma recherche doctorale (Bédard 2005). Je les comparerai principalement avec les analyses de Stillman (1995) et Gutwirth (2004), celles-ci portant sur l'ultra-orthodoxie et les Juifs d'origine marocaine, en Israël et ailleurs en diaspora. Cette comparaison m'amènera ensuite à soulever des questions d'ordre plus général en termes d'ensemble diasporique d'abord, puis par rapport à la modernité — ainsi que particulier, en regard du contexte montréalais.

\section{L'horizon sépharade}

À l'origine, l'ethnonyme «sépharade " désignait les Juifs installés dans la péninsule ibérique. À la suite de leur expulsion d'Espagne en 1492 (Wachtel 2001), par décret du royaume castillan, les Sépharades se sont réinstallés principalement sur le pourtour méditerranéen, de l'Afrique du Nord à la Palestine, la Turquie et l'Europe du Sud-Est. Ils y ont transposé leur capital culturel, intellectuel et religieux, hérité de l'Âge d'or (d'Al Andalus, $\mathrm{du} \mathrm{X}^{\mathrm{e}}$ au XII ${ }^{\mathrm{e}}$ siècles) et souvent, leur idiome judéo-espagnol, mélange de

2. Le sens de cette "tradition », tel qu'invoqué par les personnes rencontrées, est précisé plus loin. Quoique d'un même pays d'origine, le judaïsme marocain est traversé par de multiples débats, recoupant des histoires diverses (voir notamment Zafrani 1998 pour un bon aperçu). Il est donc important de ne pas substantialiser la référence à divers aspects du judaïsme marocain, fondant dès lors une référence à une tradition et gommant l'importante diversité qui prévalait au Maroc. Ce processus relève beaucoup plus d'un discours et d'une pratique, bref d'une construction de la mémoire marquée par la contemporanéité (Halbwachs 1993 ; Zerubavel 1994), par rapport à ce que nous apprend l'historiographie, plus complexe et hétérogène (Brubaker 2005). 
castillan, d'hébreu et d'arabe. Dans plusieurs cas, ils ont réussi à s'assurer une position de leadership au sein des communautés juives qui les accueillirent. Ce fut le cas au Maroc, du moins pour les grandes villes du nord (Tétouan, Tanger) et les villes impériales (Fès, Meknès). Ailleurs, les communautés locales sont demeurées davantage en retrait de l'influence de ceux qu'on appela au Maroc les Megorashim ( «expulsés »). Ainsi, ces nouveaux arrivants se sont ajoutés à la judaïcité marocaine surtout dans le nord et les grandes villes. Ailleurs au Maroc, après 1492, les Toshavim ("résidents ») demeuraient davantage à l'écart de cette influence sépharade, à des degrés variant d'une région à l'autre. Ces caractéristiques propres à la judaïcité marocaine ont eu des répercussions jusqu'à nos jours. C'est ainsi que certains Juifs marocains n'ont commencé à s'identifier à l'ensemble sépharade qu'une fois situés dans le contexte montréalais, au voisinage notamment de coreligionnaires ashkénazes.

\section{L'univers sépharade d'après 1492 et l'essor de l'hassidisme}

L'histoire du judaïsme, en particulier du mouvement ultra-orthodoxe, est traversée par de multiples courants, reflétant l'influence, elle-même hétérogène, des sociétés au sein desquelles des communautés juives se sont installées et ont développé l'ultra-orthodoxie. Je tente ici de résumer brièvement les principaux repères décrivant l'arrière-plan social et historique qui, ultimement, allait mener à l'arrivée des premières cohortes de Juifs du Maroc à Montréal, à la fin des années 1950.

La période suivant l'expulsion d'Espagne en fut une de transformation des communautés juives du pourtour méditerranéen, marquées également par ce contexte de rupture et de migration. La tradition mystique de la kabbale, inspirée notamment par l'œuvre d'Isaac Louria (1534-1572), est devenue particulièrement répandue parmi les communautés sépharades disséminées dans l'aire méditerranéenne, spécialement à Safed. C’est dans la foulée de cette effervescence mystique que s'inscrit l'épopée de Sabbataï Tsevi (1626-1676), en lequel plusieurs crurent voir le Messie. Celui-ci a rallié en particulier plusieurs communautés sépharades installées sur le pourtour méditerranéen. Dans un contexte d'attente et d'espoir messianiques, également alimenté par des massacres à la suite de la révolte cosaque en Pologne (1648-1649), la proclamation publique de son statut de Messie, lors de sa visite auprès de Nathan de Gaza en 1656, arrivait donc à un moment particulièrement propice au schème eschatologique et rédempteur de l'arrivée du Messie. La vive commotion qui a suivi son décès, 
révélant son statut de faux messie, s'est répercutée jusque dans les communautés juives d'Europe centrale et orientale, où des foyers de kabbale lourianique s'étaient développés (Cohn-Sherbok 1994, 118). L'émergence du mouvement hassidique (où hassid veut dire, littéralement, "pieux» en hébreu) se situe dans le sillon de ces espoirs brisés. Né en Podolie (région de l'Ukraine) au XVIII siècle, ce nouveau mouvement entendait — lui aussi - apporter une réponse aux difficiles conditions de vie des Juifs, cette fois du côté de l'Europe de l'Est. Durant le XIX siècle, le mouvement ultraorthodoxe prit de l'ampleur en s'opposant aux courants prônant plutôt l'intégration à la modernité: la Wissenschaft des Judentums ( «science du judaïsme») et la Haskala. Ces deux courants représentaient, au sein des communautés juives, le vent de l'intégration moderniste aux sociétés européennes, elles-mêmes formant alors leurs ensembles nationaux (Le Rider 2000). Ces courants s'avançaient alors sur les promesses de la modernité, imprimant une transformation historique au judaïsme. C'est d'ailleurs la Haskala qui allait, par la suite, constituer le courant principal à la base du sionisme, né à la fin du XIX ${ }^{e}$ siècle.

Le courant hassidique se répand en Europe centrale et orientale durant les XVIII et XIX ${ }^{e}$ siècles, à travers l'Ukraine, la Russie, la Lituanie, la Pologne et l'Autriche, principalement. Des courants divergeant sur certains points de doctrine et de philosophie prennent forme et se transmettent de génération en génération. Les communautés plus importantes ont un rebbe, leader religieux reconnu pour ses connaissances étendues et son charisme. La qualité de rebbe se transmet généralement de père en fils. Ainsi se fondent, par exemple, les dynasties Lubavitch, Breslev, Skver, Satmar, Belz, Bobov, Tash, Klausenberg, Munkatch, Pupa et Vishnitz ${ }^{3}$, du nom soit de la localité initiale où fut fondée la dynastie, soit du patronyme du premier rebbe. Au-delà de divisions basées sur l'interprétation des textes, différenciant ces différentes dynasties, le mouvement hassidique est caractérisé par sa grande piété et sa ferveur, animée d'un mysticisme associé à la tradition de la kabbale.

À Montréal, les adhérents aux courants hassidiques se trouvent à proximité de New York, où sont installées certaines des communautés les plus importantes au monde, ce qui facilite l'organisation de pèlerinages. Par exemple, les Lubavitch ont à Brooklyn leur maison-mère ${ }^{4}$, où demeure le

3. À ma connaissance, ce sont les onze branches de l'hassidisme présentes à Montréal (voir Shaffir 2001, 56, auquel j'ai ajouté les Breslev).

4. Les Lubavitch disposent d'un vaste réseau de centres Chabad, établis dans le but de permettre à tout Juif de passage de se recueillir et de se retrouver dans un contexte respectant les principes du judaïsme (la Halakha), aussi bien à Montréal qu'à Caracas ou Shanghaï (voir Brenner 2003). 
rebbe. Toutefois, cette communauté s'est retrouvée dans une situation exceptionnelle après le décès du dernier rebbe en 1994, non remplacé depuis. La vie du rebbe Schneerson a inspiré maints ouvrages et articles (voir Gutwirth 2004 pour une revue exhaustive). Dans le cadre de cet article, je me limiterai à souligner le fait que, peu avant sa mort, une partie importante des Lubavitch s'était ralliée à l'avis qu'il s'agissait du Messie. Après son décès, il y eut donc un intervalle où on évoqua même son éventuelle ressuscitation (Boyarin 1996). Certains admirent ensuite ouvertement leur erreur; plusieurs interprétèrent plutôt ce décès sans signe eschatologique comme une attente prolongée de la fin des temps. Quoi qu'il en soit, aujourd'hui à Montréal, on trouverait quelque 10000 personnes adhérant à l'ultra-orthodoxie , sur les 92970 Juifs dans la grande région de Montréal (Shahar 2003).

Les premiers Juifs d'origine marocaine à Montréal sont arrivés en 1956, au lendemain de l'indépendance du royaume alaouite qui consacrait la fin du Protectorat français. De 250000 qu'ils étaient au début des années 1950 , les Juifs montréalais d'origine marocaine ne sont plus qu'autour de 5000 aujourd'hui au Maroc, ce nombre ayant de surcroît connu une baisse de $5 \%$ par an durant les années 1990 (Lévy 1999). Si la plupart ont quitté pour Israël, plusieurs ont opté pour la France, Montréal s'avérant la troisième terre d'accueil, quoique loin derrière les deux premières. Autour de 18000 Juifs d'origine marocaine se trouvent aujourd'hui à Montréal. Ceux-ci comptent pour près de $80 \%$ des Sépharades montréalais. Ils sont également majoritaires parmi les écoles juives francophones, que fréquentent la plupart des enfants des familles d'origine marocaine ${ }^{6}$. Il s'ensuit que la plupart des jeunes d'origine marocaine grandissent et sont socialisés dans un milieu fortement marqué par l'origine marocaine des parents, religieusement et culturellement. Enfin, suite logique à cet exode massif et rapide

5. Un sondage de 1997 indique une population «hassidique et ultra-orthodoxe » d'environ 6250 personnes à Outremont et ses environs (Shaffir 2002, 69). De plus, les Tash de Boisbriand forment une communauté de quelque 3000 personnes (Shaffir 2000, 76). C'est ainsi que Shaffir $(2001,56)$ arrive au chiffre approximatif de 9250.

6. On trouve peu de données fiables pour préciser cette affirmation. Néanmoins, toutes les observations dégagées lors de ma recherche de terrain abondent dans ce sens. De plus, déjà avec Elbaz (1994), on voit que dans les années 1980, 63 \% des jeunes Sépharades fréquentaient le réseau d'écoles juives. L'évolution depuis favoriserait une tendance plutôt à la hausse qu'à la baisse de ces effectifs, y compris une augmentation des milieux plus orthodoxes. Ceci est corroboré, entre autres, par la création en 1994 de l'AcadémieYeshiva Yavné, dont le nombre d'élèves, depuis, a crû de façon importante. 
du Maroc, les Juifs montréalais d'origine marocaine ont pratiquement tous des proches en Israël, plusieurs en ayant également en France.

\section{Parcours familiaux et reconstructions de la mémoire}

Mon analyse des constructions de la mémoire s'appuie d'abord sur des entrevues menées entre 2000 et 2002 auprès de 23 personnes, issues de 16 familles, couvrant trois générations de Juifs d'origine marocaine. La première génération est née entre 1915 et 1930, la génération médiane, entre 1940 et 1955 et la génération cadette, entre 1965 et 1980 . La première a connu le Maroc colonial, quittant le Maroc soit avec leurs enfants, soit les suivant dans la migration à Montréal. La seconde génération a quitté le Maroc au début de l'âge adulte, parfois avant. Quant à la génération cadette, elle est presque entièrement née à Montréal, sinon arrivée en bas âge. Leur mémoire du Maroc passe donc par ce que leur auront transmis (ou non) leurs parents et grands-parents. Il est aussi intéressant de noter qu'un tiers des Juifs d'origine marocaine venus s'installer à Montréal a auparavant effectué un séjour en Israël (Elbaz 1989, 92). Enfin, notons qu'à ces entrevues se sont ajoutées des observations lors de célébrations religieuses et communautaires, des conversations et des entrevues avec des informateurs clés.

L'analyse de ces données m'a permis de dégager une catégorie principale de rediasporisation ${ }^{7}$, que j'ai appelée traditionaliste, relevée chez sept des seize familles rencontrées. Les récits présentent principalement des événements datant de la fin du XIX ${ }^{e}$ siècle à aujourd'hui. En relatant leurs parcours personnels et leurs histoires familiales, ces individus montrent un itinéraire social où la plupart des membres de leurs familles sont demeurés près d'une pratique régulière du judaïsme. Toutefois, les propos recueillis insistent souvent sur les valeurs centrales de souplesse et de tolérance, affichées comme des qualités intrinsèquement sépharades. Du même souffle, ils font ressortir une distanciation face aux mouvements ultra-orthodoxes. Leur intransigeance et leur rigueur sont perçues comme des dimensions auparavant absentes de l'horizon de ces familles, au Maroc même ou durant les premiers temps de l'immigration à Montréal. Quant à la plus grande ferveur religieuse associée aux Juifs hassidiques, elle n'est la plupart du temps évoquée qu'à demi-mot, de façon ambiguë. En les désignant comme «les

7. À l'instar d'Elbaz (1994), je parle de rediasporisation, puisque les Juifs au Maroc se trouvaient déjà dans une situation diasporique. Ils ont ainsi connu une rediasporisation en quittant le Maroc pour s'installer ailleurs qu'en Israël, comme à Montréal. 
barbus » ou en mentionnant certaines de leurs pratiques comme des «superstitions » auxquelles ils n'adhèrent pas, ces personnes d'une religiosité empreinte de cette "souplesse sépharade » prennent une position aussi critique que possible, sans franchir le point où il s'agirait d'une position clairement négative envers ces mouvements et sans préciser davantage ces brèves allusions. Les commentaires à l'égard des ultra-orthodoxes sont donc demeurés polis et réservés. Tout juste évoquait-on ces mouvements, de plus en plus présents dans le paysage de familles d'origine marocaine, sur un mode chevauchant l'empathie et l'agacement.

Tout au long de la recherche, on m'a parlé de familles où certains jeunes adultes avaient décidé d'adhérer à l'un ou l'autre des mouvements ultraorthodoxes. La conséquence la plus souvent mentionnée dans de telles situations est le refus, par ces jeunes adultes, de venir manger chez leurs propres parents. Après l'adhésion à l'ultra-orthodoxie, leurs normes (notamment culinaires) faisaient en sorte qu'à leurs yeux, le foyer de leurs parents était devenu insuffisamment cachère. Plusieurs familles ayant participé à ma recherche remerciaient Dieu que ce ne soit pas leur situation. C'était le cas, néanmoins, pour une de ces familles. Là, pour cet homme dont le frère aîné, dans la cinquantaine, s'était rallié à un mouvement ultra-orthodoxe, cela ne provoquait pas de tensions aussi épineuses; il soulignait même que son frère était "demeuré sépharade». Quels étaient ces éléments sépharades qui demeuraient, malgré une pratique devenue ultra-orthodoxe? C’est qu'il reconnaissait dans l'ultra-orthodoxie de son frère des éléments correspondant à certains traits de la vie traditionnelle juive qu'on pouvait trouver au Maroc. Il y voyait en effet une continuité à travers le fait que toutes les dimensions de la vie de son frère étaient, comme dans la vie traditionnelle au Maroc, marquées par son adhésion à l'ultra-orthodoxie:

C. Benattar (pseudonyme) - Non, non, il reste sépharade, mais il a un rythme... Même chez les Sépharades, il y en avait qui étaient très religieux, mais pas du mouvement hassidique. Tu sais, la religion au Maroc, chez les Juifs, ça réglait tout. Le shabbat réglait tout, les fêtes réglaient tout, parce que n'oublie pas que chez les Juifs, c'est une fête après l'autre, hein! Mais c'était... ça réglait tout. Ça réglait le temps! Le temps était ponctué de fêtes, de shabbat, c'était comme ça. C'était naturel, pas de façon extrémiste, mais c'était normal. C'était la normalité de la vie, le shabbat, c'est normal que tous les Juifs fassent le shabbat au Maroc!

Retenons donc cette association entre la normalité au Maroc et l'adhésion à l'ultra-orthodoxie dans le contexte contemporain, en rediasporisation à Montréal. Si mes interlocuteurs faisaient généralement preuve de 
réserve dans leurs propos concernant l'ultra-orthodoxie, ce n'était pas le cas d'un jeune homme dans la vingtaine. Celui-ci m'a fait part de son point de vue quant à l'origine de l'attrait pour l'ultra-orthodoxie parmi les jeunes d'origine marocaine, ainsi que son impact sur la séphardité:

N. Chitrit — Là où ça me dérange, c'est que les mouvements hassidiques, surtout les Lubavitch, ont une espèce de méthode de outreach [...]. Ils ont essayé beaucoup chez les Ashkénazes, ici à Montréal, ça marche pas. [...] Les Sépharades sont très... par leur nature, par leur culture qui s'est développée, beaucoup plus réceptifs à tout ce qui est de l'ordre du superstitieux, du surnaturel... justement, du tsadik [litt. «saint»; personne pieuse, d'une grande sagesse] [...]. Et il se trouve que les Sépharades sont assez réceptifs à ces messages justement non seulement du tsadik, mais aussi ces messages aussi un peu messianiques... Je dis pas que je crois pas dans le Messie, [...] mais [la notion de Messie à laquelle j'adhère] est très différente de celle que les Lubavitch nous présentent...

Tout ça pour dire que ces mouvements, je vois ça négativement, parce que ça me dérange de voir du jour au lendemain un Sépharade passer d'une certaine façon de s'habiller, une certaine façon de se comporter, au chapeau noir et à l'habit traditionnel hassidique, qui est très bien! tant que c'est dans leurs traditions [...]. Donc, ça serait dommage de laisser tomber cet aspect sépharade, qui est très... une culture tellement forte, tellement riche! [...] Ça existe, ce grugeage! comme je dis, parce qu'on est beaucoup plus prompts à se faire embarquer dans ces mouvements, que les Ashkénazes qui sont là ça fait 150 ans et qui ont une certaine rationalité peut-être différente.

D'autres commentaires critiques ont été entendus durant ma recherche, venant cette fois de personnes issues d'autres familles que celles associées à la rediasporisation traditionaliste, mentionnée auparavant. Parmi ces familles, j'ai plutôt relevé des discours et des positions sociaux se situant volontairement en périphérie par rapport au milieu sépharade et juif. Tout en exprimant une identité juive importante, ces personnes ont pris leurs distances d'une religiosité plus traditionnelle. Certaines ont même en aversion le phénomène religieux, d'une façon semblable à ce qu'on peut trouver parmi la population québécoise francophone (des suites de la Révolution tranquille). Par exemple, chez la famille Oliel, l'aînée de la famille, elle-même arrivée à Montréal après son fils, dans les années 1960, qualifiait de «folie» la pratique plus rigoureuse des hassidim:

J.-L. Bédard - Est-ce que vous avez connu un milieu assez pratiquant, quand vous étiez jeune? [N.B. Son père était rabbin.] 
C. Oliel — Chez mes parents ? Ah oui! Absolument. Absolument... mais pas comme... pas comme les hassid qu'il y a maintenant ici... Non, on n'a pas été élevés comme ça, non. On a été élevés avec... avec la religion, comme tout le monde. [...] Mais... vous savez, notre religion, c'est un peu des coutumes, un peu des... comment dirais-je... pas la religion trop rigide. Voilà. Non. Mon père était très libéral. Très libéral. On n'a pas été élevés dans... comme ici, je vois, c'est la folie. La folie.

Un peu plus loin, elle commente sa religiosité tout comme plusieurs Juifs d'origine marocaine de sa génération: «notre religion, on la pratique, suivant nos possibilités, suivant nos idées! Et c'est tout! Sans excès, trop fort, ces excès, à quoi ça sert ? Ça sert à rien! » Son fils, que j'ai rencontré par la suite, a marié une Québécoise francophone. Depuis l'enfance, il a été loin de toute pratique religieuse et n'a pas été tenté, plus tard, de s'en rapprocher. Plus loin dans l'entrevue, il précise que cet éloignement de la tradition juive est le prolongement de l'influence de son père. Après l'immigration à Montréal, il a même développé une aversion pour le milieu ultra-orthodoxe et ce qu'il qualifie d'intégrisme juif. Il le vit dans sa famille même, puisque chez un oncle en France, les enfants sont devenus ultra-orthodoxes:

A. Oliel - Mon oncle lui-même, qui est quand même... qui est fils de rabbin, qui est plus religieux, il va à la synagogue le samedi, et tout, mais lui, pour lui, ses enfants, qu'ils soient devenus orthodoxes comme ça, pour lui c'est un désastre! Dans sa vie à lui... Pour lui, c'est comme... une insulte à ce qu'il est, lui. C'est... c'est terrible!

\section{Séphardisation, ashkénazisation : même perspective moderniste}

La pratique rigoureuse prônée par les divers mouvements hassidiques est d'origine ashkénaze. C'est lors de la création de ces mouvements, en Europe de l'Est des XVIII et XIX siècles, qu'ont été instaurées ces pratiques caractérisées par des règles d'hygiène très strictes et la minimisation des contacts avec la société environnante, notamment par la structure de l'enclave (Almond et coll. 2003). Historiquement, on ne trouve pas d'équivalent parmi les Sépharades, que ce soit dans les pays arabes - où la plupart des Sépharades se trouvaient jusque vers les années 1950 - , en Europe ou en Amérique. Ainsi, d'un point de vue historique ne prenant en compte que les événements ayant eu lieu depuis l'expulsion de 1492, on peut qualifier d'influence ashkénaze l'adhésion de Sépharades à des mouvements ultra-orthodoxes. Pourtant, on voit tour à tour Stillman (1995) parler d'ashkénazisation 
parmi des Juifs d'origine marocaine et Gutwirth (2004) de séphardisation parmi des congrégations ultra-orthodoxes. Examinons d'abord leurs analyses, pour ensuite les commenter et proposer une autre analyse, plus générale, intégrant ces mouvements dans la longue durée.

D'abord, à partir du contexte israélien, Stillman (1995) a parlé d'une ashkénazisation des Juifs de provenance marocaine à travers leur adhésion à des courants hassidiques d'origine ashkénaze. Surtout, il invoque un rigorisme et une rigidité croissante sur le plan des pratiques pour fonder son constat d'ashkénazisation, s'empressant toutefois de souligner qu'il ne s'agirait que de groupes aux nombres limités. Il constate la mise de côté de la tradition sépharade de souplesse et de flexibilité, dimensions centrales également évoquées à maintes reprises lors de ma recherche. De son côté, Gutwirth $(2004,179)$ analyse les mouvements hassidiques en France et souligne à quel point ceux-ci se sont "séphardisés », processus qu'il a relevé en particulier à Paris par plusieurs indices (organisation physique des synagogues, nature des célébrations à certaines fêtes, etc.). L'hospitalité et la tolérance sont des valeurs sur lesquelles se rejoignent les traditions culturelles juives nord-africaines et hassidiques Lubavitch. C'est sur la base de ces différents aspects que Gutwirth parle ainsi d'une séphardisation de l'hassidisme Chabad en France.

Conséquemment, de quel côté est-ce que l'ensemble penche? Devraiton parler d'ashkénazisation à un endroit, de séphardisation ailleurs? Ce qui nous intéresse surtout ici, c'est de répondre à la question suivante: comment devrait-on qualifier l'attrait de Juifs montréalais d'origine marocaine envers ces mouvements ultra-orthodoxes? Comment pourrait-on mieux l'expliquer? Est-ce que ceux-ci viennent "séphardiser» un mouvement a priori ashkénaze, ou subissent-ils plutôt une influence ashkénaze? Une option serait d'arriver à une description éclatée de la dynamique des Juifs d'origine marocaine, en Israël et dans les divers sites de la diaspora. On observerait donc une séphardisation à Paris (ce que relève Gutwirth) et une ashkénazisation en Israël (d'après Stillman). Quant à Montréal, s'agirait-il d'une ashkénazisation ou d'une séphardisation? Il ne faut pas oublier que vu l'exode récent du Maroc, à Montréal ou ailleurs en diaspora, les liens entretenus avec des membres de la famille sont encore très présents, alimentés par les visites de part et d'autre, à l'occasion de mariages ou de bar-mitsvah. Il y a donc une circulation importante des personnes et des idées entre les divers sites de la diaspora (dont Montréal) et Israël. Face à ces constats soulignant l'identification tantôt à l'un, tantôt à l'autre groupe (sépharade 
et ashkénaze), chacun posé comme distinct, je propose plutôt de questionner une perspective toujours présente dans ces catégorisations. Il s'agit de l'accent sur le lignage ou la généalogie immédiats, au détriment de l'analyse du rapport au passé et de la construction de la mémoire du groupe. En examinant ces dernières dimensions, on se rend compte qu'elles mettent en scène, dans ce milieu sépharade, mais avant tout juif, un rapport au passé permettant d'enjamber d'importantes périodes historiques. Concrètement, cela se traduit par la proximité de modes d'interprétation de l'histoire hérités du Moyen Âge, véhiculés par le paradigme religieux, en particulier à travers la kabbale lourianique, qui a peu à faire des interprétations historiques «modernes».

Mes données à ce sujet, limitées, nécessitent l'appui d'autres sources pour fonder mon argumentation ${ }^{8}$. L'analyse du rapport au passé dans le monde juif menée par Yerushalmi (1984) nourrit le même argument. On y voit que le rapport au passé traditionnel des milieux juifs est caractérisé par l'espoir messianique et l'interprétation issue de la kabbale, et l'approche historiographique moderne n'y a que peu de place:

Les Juifs qui se tiennent encore à l'intérieur du cercle enchanté de la tradition, ou ceux qui y sont retournés, nient toute pertinence au travail de l'historien. Ce qu'ils cherchent dans le passé, ce n'est pas son historicité, mais son éternelle contemporanéité. Posée directement par le texte, la question de savoir comment le passé évolua doit leur sembler subsidiaire, sinon dénuée de sens. (Yerushalmi 1984, 113)

Des propos entendus au lendemain des attentats du 11 septembre 2001, lors d'une des principales célébrations du calendrier juif, abondent dans ce sens. Un interlocuteur y mentionna que d'après plusieurs rabbins jouissant d'une certaine autorité dans le milieu ultra-orthodoxe, l'horizon de l'an 6000 du calendrier juif donnait la date approximative à laquelle le

8. J'expose les raisons de ces limites dans ma thèse (Bédard 2005). Brièvement, c'est parmi ces milieux plus près de l'ultra-orthodoxie que j'ai essuyé le seul refus explicite de participation à ma recherche. Ce refus s'ajoute aux autres difficultés rencontrées dans ces milieux, que j'ai interprétées comme des refus implicites (appels non retournés, annulations de rendez-vous à la dernière minute, etc.). Il en résulte que les personnes ayant accepté et effectivement pris part à la recherche furent, par rapport au total de celles sollicitées, largement minoritaires, en particulier parmi celles qu'on peut associer à la mouvance ultra-orthodoxe. Ces difficultés ont déjà été relevées de façon plus exhaustive par d'autres, notamment Shaffir (2001). 
Messie reviendrait (nous sommes présentement en 5767) 9 . Cet interlocuteur insistait sur l'importance de préparer cette venue par la techouvah (acte de retour à la foi et à la pratique du judaïsme), dans l'esprit du tiqqun (travail de «réparation" dans l'équilibre de l'Univers, qui mène à la rédemption). Il ajoutait que la Shoah d'abord, puis le 11 septembre 2001 étaient des événements dont la gravité ne faisait que confirmer l'imminence de cette date, de plus en plus proche (moins d'un quart de millénaire). Ces propos ont été entendus dans une congrégation où on peut noter la présence des Lubavitch. Gutwirth (2004) mentionne à leurs propos qu'après avoir cru que leur rebbe était le Messie, malgré son décès et les années qui passent, cette croyance perdure. Les Lubavitch sont reconnus pour leur croyance en la capacité d'intercession par le biais de l'action humaine, celle-ci servant alors à précipiter l'avènement du Messie et la fin des temps (Almond et coll. 2003, 63). Ceux qui renouent avec la pratique du judaïsme, les baal techou$v o t$, participent de ce mouvement qui vise précisément à accélérer le cours eschatologique de cette histoire. C'est pourquoi, peut-on penser, cet homme soutenait que l'horizon de l'an 6000, à plus de deux siècles d'aujourd'hui, serait en fait raccourci, d'autant plus par la pratique fervente des baal techouvot dont le nombre croissant viendrait accélérer ce mouvement de rédemption et rapprocher l'imminence de la fin des temps. Comme le mentionnent Almond, Appleby et Sivan (2003), les mouvements fondamentalistes ont tendance à se présenter comme comptant de plus en plus d'adhérents. La nature multisituée de ces groupes rend difficile un suivi précis du nombre réel d'adhérents. Leur nombre croissant pourrait être vrai localement sans que cela soit le cas globalement. Bref, on assiste là aussi (comme à propos de références à «la tradition ») à davantage de rhétorique et de présentation stratégique qu'à un relevé objectif.

9. Ici aussi, il y a plus d'une position à cet égard. Selon la lecture que fait Ouaknin (1993) de l'œuvre de rabbi Nahman de Breslev, le temps messianique n'y est pas:

...le temps où le Messie serait là. Au contraire: c'est le temps où le Messie est attendu. Pour forcer le trait: le Messie est fait pour ne pas venir... et, pourtant, il est attendu. [...] L'homme messianique (celui qui attend) se projette constamment dans l'à-venir du futur, il produit de la différence, du suspens. [...] Dans cette mise en suspens du temps, le temps est toujours jeté vers l'à-venir par un acte d'anticipation. Mais l'anticipation ne prévoit rien; il n'y a pas d'accomplissement au bout du chemin. Il y a anticipation d'anticipations. C'est en ce sens que R. Nahman dit: "Tous mes enseignements ne sont que des introductions... » (Ouaknin 1993, 412-413). 


\section{Rapports au passé et catégories ambiguës}

De plus, l'analyse des constructions de la mémoire, mentionnées durant ma recherche à Montréal, permet de voir que les rapports au passé parmi des familles plus près des mouvements ultra-orthodoxes sont moins marqués par la modernité et semblent plus près du rapport au passé traditionnel du milieu juif ${ }^{10}$. Dans la perspective de ce rapport au passé, les distinctions sépharades-ashkénazes et les influences que jouent l'un et l'autre ensemble s'entremêlent. En effet, lorsqu'on remonte en amont, au Moyen Âge, on voit qu'il y avait influences réciproques et circulations. Pour autant, cela n'invalide pas toute distinction entre ces deux groupes. Autrement dit, il n'en découle pas que toute différence en termes de ces critères est, finalement, dénuée de fondements. Plutôt, il apparaît important de rappeler, à la lumière de l'analyse présentée ici, que ces catégories mêmes de «traditions sépharades » et «traditions ashkénazes » sont des distinctions qui ne sont pas mutuellement exclusives, hier comme aujourd'hui. Bien sûr, ces univers étaient autrefois davantage séparés géographiquement. Néanmoins, ils se sont depuis longtemps interpénétrés, tout en maintenant d'importants éléments distinctifs, liés à leurs ancrages culturels respectifs. Certes, la question devient plus complexe aujourd'hui, étant donné la circulation des personnes, des idées et des capitaux, plus importante que jamais dans l'histoire. Il faut toutefois se rappeler qu'aussi bien durant l'Antiquité qu'au Moyen Âge, en particulier dans la diaspora juive, les personnes, les idées et les capitaux circulaient également, quoiqu'à un rythme plus lent qu'aujourd'hui (Attali 2002; Wachtel 2001; Wasserstein 1996). Néanmoins, il

10. J'ai illustré ce point dans ma thèse (Bédard 2005) par davantage d'éléments que les quelques-uns présentés ici, dont la démonstration serait trop longue à reprendre dans le cadre d'un article. Essentiellement, je montre que la nature du contact avec la modernité, dès le Maroc, a eu un impact sur le parcours migratoire, l'insertion socioprofessionnelle et la socialisation des enfants à Montréal. Les familles ayant eu un contact plus étroit avec l'appareil colonial français et le système scolaire de l'Alliance israélite universelle, notamment, ont connu une meilleure insertion socio professionnelle à Montréal. Leurs enfants ont été en grande majorité socialisés dans un cadre juif sépharade et francophone. Parmi les deux familles où de jeunes adultes sympathisent avec les courants ultra-orthodoxes, les parents étaient plus éloignés de l'appareil colonial et des institutions juives françaises au Maroc. Quoique réussie, leur insertion socio-professionnelle à Montréal s'inscrit davantage dans les métiers et le commerce que les professions libérales. À leur tour, consacrant cette trajectoire, leurs enfants ont connu une socialisation dans le milieu juif, certes, mais plus près du milieu ashkénaze et anglophone (sur le plan de la scolarisation notamment). 
y avait circulation à travers la diaspora. Les figures marranes de Spinoza, Montaigne et Thérèse d'Avila témoignent également de la circulation d'idées issues de la culture juive et sépharade dans la société européenne et chrétienne. Aux fins de cet article, j'ai retenu le parcours du faux messie Sabbataï Tsevi, qui allait ensuite contribuer, au côté d'autres facteurs, à l'apparition des mouvements hassidiques en Europe de l'Est, dans le contexte consécutif à l'expulsion des Sépharades en 1492. Il s'agit là d'une illustration, au cœur de la sphère religieuse, de la circulation d'idées notamment, de voies de communication entre les univers sépharades et ashkénazes. On peut voir des conséquences de cet entrecroisement jusqu'à nos jours, entre autres à Montréal, que ce soit à travers les constructions de la mémoire ou l'analyse qui en découlent, ce que j'ai tenté de démontrer ici par des données de ma recherche.

D'un point de vue plus synchronique, on peut également s'interroger sur les conséquences de la coprésence, à Montréal, parmi le milieu juif sépharade, de courants ultra-orthodoxes et de courants "traditionnels» qui, comme on l'a vu, sont empreints d'une souplesse d'autant plus importante qu'elle va de pair avec "la tradition », associée à la séphardité. Il en découle une polarisation entre ceux qui adhèrent aux mouvements ultraorthodoxes - quelle que soit la séphardisation que ceux-ci impriment à leurs congrégations ou inversement, l'ashkénazisation qu'on y retrouve et ceux qui vivent un judaïsme sépharade «traditionnel », c'est-à-dire empreint de modération, de souplesse ainsi que de ferveur. Cette polarisation atteint des dimensions encore plus marquées avec les individus qui pratiquent peu ou (rarement) pas du tout. Comme je le mentionnais en introduction, peut-être le seul aspect commun au sein de cette diaspora à structure chaordique demeure l'attachement envers l'existence et le maintien de l'État d'Israël. Dans ce contexte, les individus peu pratiquants ressentent une grande distance les séparant du milieu juif montréalais (la communauté instituée), identifié comme un des plus conservateurs en Amérique du Nord, en particulier dans le domaine religieux (Shahar et Schnoor 1997).

Sur le plan méthodologique, il apparaît important de souligner que l'usage des catégories regroupant des collectivités, dans ce cas-ci «sépharades » et "ashkénazes", doit être fait en tenant compte de l'histoire des relations entre celles-ci. L'analyse permet donc de voir que ce qui semble tout à fait surprenant - l'attrait de jeunes Montréalais juifs d'origine marocaine envers certains mouvements hassidiques (dont les Lubavitch) - ne l'est pas tant qu'à première vue, lorsqu'on considère la longue durée. Ceci est 
facilité par des cadres sociaux qui favorisent, de mille et une façons, un rapport vivant à un passé lointain, du moins chronologiquement (Hobsbawm et Ranger 1983; Yerushalmi 1984). Tout comme les moyens de communication permettent d'effacer, du moins symboliquement, les distances physiques, ces constructions de la mémoire permettent de gommer des distances temporelles importantes, leur donnant une contemporanéité par l'intermédiaire de rapports au passé différents de ceux généralement mis de l'avant par la modernité (Yerushalmi 1984; Almond et coll. 2003).

\section{Conclusion}

La thèse de la modernité liquide (Bauman 2000) insiste sur le caractère mouvant des identités contemporaines et l'importance de considérer le passage du temps, de façon précise étant donné la plus grande labilité des constructions contemporaines. Cette métaphore peut s'appliquer en termes généraux et Bauman poursuit sa réflexion pour en démontrer des conséquences dans la nature de plus en plus transitoire et éphémère des relations interpersonnelles, dans les sphères du travail et même de la famille (2004). En contrepartie, cette fluidité apparaît peu éclairante pour comprendre l'hétérogénéité et la multiplicité des courants se côtoyant au sein d'un même ensemble diasporique, tel qu'on l'a vu ici avec des Juifs d'origine marocaine à Montréal. En d'autres termes, les rapports relativement hermétiques entre les divers courants relèvent de frontières internes au groupe diasporique, davantage que de frontières entre le groupe et la société globale, ce qui ramène à l'avant-plan les travaux de Barth (1969) sur la dynamique de construction des frontières. Celle-ci paraît fondamentale pour la construction de l'identité et de la définition de soi et des autres, en fonction du contexte par lequel des groupes se constituent, en réaction à leur coprésence. Ces frontières ne limitent pas tant la fluidité des échanges qui circulent, autrement dit la morphologie de ces échanges, comparables à ce niveau aux échanges auxquels on assiste à l'échelle de la société globale, québécoise en l'occurrence. La différence réside dans les canaux empruntés par ces échanges, ouverts - ici dans un milieu juif, a fortiori parmi des mouvements ultra-orthodoxes - de façon prioritaire sur des voies transnationales relayant des constructions de la mémoire se démarquant, comme on l'a vu, des schèmes modernistes, plus près de l'historiographie moderne. Ces échanges, canalisés à travers l'établissement de frontières caractérisés par une cohérence interne, créent les conditions favorables au maintien d'un groupe diasporique, voire à l'accroissement de sa propre diversité 
interne, tout en négociant son inscription dans la société d'accueil, dans ce cas-ci montréalaise. Il y a donc homologie entre la notion de cadres sociaux de la mémoire (Halbwachs 1993) et celle de frontière chez Barth (1969), à tout le moins dans l'analyse du processus de représentation de soi et des autres, tel que j'ai cherché à l'illustrer à partir de ce que j'ai pu observer parmi des Juifs d'origine marocaine à Montréal. Dans sa "mise en scène ", cette diversité se trouve facilitée par la présence de cadres sociaux qui marquent ces différences par leurs rapports spécifiques au temps et à l'historicité.

Ces remarques soulignent, une fois de plus, l'importance de la relativité des catégories identitaires et la capacité des individus — des acteurs à s'en jouer, parfois à leur insu, imprimant alors des distorsions dans les usages. Ainsi, si l'adhésion à des mouvements ultra-orthodoxes peut surprendre de prime abord, l'analyse des constructions de la mémoire et surtout la considération de la longue durée permettent de voir comment le rapport au passé met en scène une tout autre représentation de soi et des autres.

En termes de perspectives d'avenir, l'attrait de mouvements ultraorthodoxes parmi de jeunes Juifs montréalais d'origine marocaine semble s'inscrire dans le mouvement plus vaste de l'attrait exercé par les mouvements fondamentalistes, tel que relevé par Almond, Appleby et Sivan (2003). Au-delà de ce constat général, on aura vu toutefois que cet attrait s'articule à travers le croisement implicite de catégories posées comme plus distinctes (sépharades, ashkénazes) qu'elles ne le sont en réalité, "oubliant » au passage leurs fondements communs. A posteriori, on peut comprendre cet entrecroisement de mémoires et de processus d'identification en lien avec les conditions actuelles de rediasporisation des Juifs d'origine marocaine à Montréal, de surcroît au côtoiement d'un milieu juif ashkénaze, dont une partie est composée de plus d'une dizaine de mouvements hassidiques. Ce voisinage est loin d'être unique à Montréal; il ne constitue en fait qu'un pâle reflet du côtoiement, plus intense, vécu par ces groupes et d'autres en Israël. En considérant l'inscription de ces jeunes adultes dans la vie montréalaise, ceci met en exergue, du reste, le côtoiement de groupes de citoyens avec des horizons fondamentalement différents. Les dérives récentes dans l'actualité québécoise autour de la pratique, juridiquement encadrée, des accommodements raisonnables, illustrent le fait qu'il devient urgent de débattre de ces horizons, afin de continuer à identifier les terrains d'entente nécessaires au vivre ensemble, la convivencia bien connue historiquement des Sépharades. Ces propos débordent toutefois du sujet de cet article; il s'agit en quelque sorte d'un versant en aval, découlant de l'analyse de l'attrait de l'ultra-orthodoxie présentée ici. 


\section{Références}

Almond, G.A., R.S. Appleby et E. Sivan (2003), Strong Religion, Chicago / Londres, University of Chicago Press.

Attali, J. (2002), Les Juifs, le monde et l'argent, Paris, Fayard.

BARTH, F. (1969), «Introduction», dans F. BARTH, dir., Ethnic Groups and Boundaries. The Social Organization of Culture Difference, Londres, George Allen \& Unwin, p. 9-38.

Bauman, Z. (2000), Liquid Modernity, Cambridge, Polity Press. Polity Press.

BÉDARD, J.-L. (2005), Se souvenir, dire, devenir. Constructions de la mémoire chez des générations de Sépharades à Montréal, thèse de doctorat, Québec, Université Laval.

BeInART, H.M. (1971-1973), «Sephardim », dans Encyclopaedia Judaica, p. $1164-1177$.

Boyarin, J. (1996), Thinking in Jewish, Chicago, Chicago University Press.

BRENNER, F. (2003), Diasporas, Paris, Martinière.

Brubaker, R. (2005), "The «Diaspora» Diaspora ", Ethnic and Racial Studies, 28/1, p. 1-19.

COHN-SHerbok, D. (1994), Atlas of Jewish History, Londres / New York, Routledge.

Elbaz, M. (1989), "D’immigrants à ethniques: analyse comparée des pratiques sociales et identitaires des Sépharades et Ashkénazes à Montréal ", dans J.-C. LASRY et C. TAPIA, dir., Les Juifs du Maghreb. Diasporas contemporaines, Montréal / Paris, Presses de l'Université de Montréal / L'Harmattan, p. 79-101.

(1994), «Ethnicité et générations en Amérique du Nord. Le cas de la seconde génération de Juifs sépharades à Montréal ", Revue internationale d'action communautaire, 31/71, p. 63-77.

GuTwirTh, J. (2004), La renaissance du hassidisme, Paris, Odile Jacob.

Halbwachs, M. (1993), Les cadres sociaux de la mémoire, Paris, Presses universitaires de France.

Hobsbawm, E. et T. Ranger (1983), The invention of Tradition, Cambridge, Cambridge University Press. 
Le Rider, J. (2000), Modernité viennoise et crises de l'identité, Paris, Presses universitaires de France.

LÉvY, A. (1999), "Playing for Control of Distance: Card Games between Jews and Muslims on a Casablancan Beach ", American ethnologist, 26/3, p. 632-653.

OuAKnin, M.-A. (1993), Le Livre brûlé, Paris, Lieu commun.

Shaffir, W. (2000), "Hassidim and Their Rebbe: Some Initial Observations ", Jewish Journal of Sociology, 42/1-2, p. 73-85. (2001), «Fieldwork among Hassidic Jews : Moral Challenges and Missed Opportunities », Jewish Journal of Sociology, 43/1-2, p. 53-69.

(2002), «Outremont's Hassidim and Their Neighbours : An Eruv and Its Repercussions ", Jewish Journal of Sociology, 44/1-2, p. 56-71.

Shahar, C. (2003), The Jewish Community of Montreal, Part I. Basic Demographics, Montréal, Federation CJA (2001 Census Analysis Series), document non publié.

Shahar, C. et R. Schnoor (1997), A Survey of Jewish Life in Montreal. Part II, Montréal, Federation CJA, document non publié.

Stillman, N.A. (1995), Sephardi Religious Responses to Modernity, Luxembourg, Harwood Academic Publishers.

Wachtel, N. (2001), La foi du souvenir. Labyrinthes marranes, Paris, Seuil (La Librairie du XXI ${ }^{\mathrm{e}}$ siècle).

WassersteIn, B. (1996), Vanishing Diaspora. The Jews in Europe since 1945, Cambridge, Harvard University Press.

Werbner, P. (2001), "The Place which Is Diaspora: Citizenship, Religion and Gender in the Making of Chaordic Transnationalism ", Journal of Ethnic and Migration Studies, 28/1, p. 119-133.

Yerushalmi, Y.H. (1984), Zakhor. Histoire juive et mémoire juive, Paris, La découverte.

Zafrani, H. (1998), Deux mille ans de vie juive au Maroc. Histoire et culture, religion et magie, Paris, Maisonneuve et Larose.

Zerubavel, Y. (1994), «The Historic, the Legendary, and the Incredible: Invented Tradition and Collective Memory in Israel ", dans J.R. GILLIS, dir., Commemorations. The Politics of National Identity, Princeton, Princeton University Press, p. 105-123. 


\section{Résumé}

Mon analyse porte sur des constructions de la mémoire et de l'identité, parmi trois générations de Juifs montréalais d'origine marocaine. En particulier, les rapports avec les mouvements ultra-orthodoxes sont révélateurs de positions quant à la judéité et à la séphardité, dans un processus de différenciation par rapport aux coreligionnaires ashkénazes. À travers une analyse du rapport au passé et des constructions de la mémoire, en plus de l'historiographie des rapports entre Ashkénazes et Sépharades depuis l'expulsion d'Espagne de 1492, il en découle une remise en question, partielle, de ces deux catégories ethnoreligieuses. Comme les cadres sociaux de la mémoire, la notion de frontière permet d'éclairer les processus contemporains de représentation de soi et des autres, parmi cet ensemble diasporique qui négocie ainsi, à la fois, ses rapports à la société locale, ses frontières internes et ses dynamiques transnationales.

\section{Abstract}

My analysis examines memory and identity constructions among three generations of Montreal Jews of Moroccan origin. Emphasis is placed on opinions towards ultra-orthodox movements which reveal positioning of their own Jewish and Sephardic identity, in a differentiating process from their Ashkenazim fellow Jews. Analysis of relations to the past and memory constructions, as well as the historiography of relations between Ashkenazim and Sephardim since the 1492 expulsion from Spain, results in a partial questioning of these two ethno-religious categories. Like memory's social frames, the notion of frontier sheds light on contemporary processes of representations of self and others, inside this diasporic entity, therefore negotiating, at once, its relations with the local society, its internal frontiers and its transnational dynamics. 